\title{
Nuclear astrophysics with recoil mass separators: ERNA and the case of ${ }^{12} \mathrm{C}(\alpha, \gamma){ }^{16} \mathrm{O}$
}

\section{Gialanella ${ }^{1}$}

Dipartimento di Scienze Ambientali, Seconda Università di Napoli, Caserta, Italy and INFN-Sezione di Napoli, Italy

Via Vivaldi 43, Caserta, Italy

E-mail: lucio.gialanella@na.infn.it

\section{Schürmann ${ }^{1}$}

Dipartimento di Scienze Fisiche Università Federico II , Napoli, Italy and INFN-Sezione di Napoli, Italy Via Cintia snc, Napoli, Italy

E-mail: daniel.schuermannena.infn.it

The use of Recoil Mass Separators (RMS) for the direct detection of recoiling ions produced in radiative capture reactions offers the opportunity to study nuclear processes of astrophysical interest in a nearly background free condition. During the last decade, several efforts were started, aiming to the refinement of this methodology. In a series of two lectures at the European School on Nuclear Astrophysics at S. Tecla, Italy, the working principles of RMS's were reviewed and some applications described. In particular, the apparatus ERNA (European Recoil separator for Nuclear Astrophysics) and some recent achievements in the study of the key reaction ${ }^{12} \mathrm{C}(\alpha, \gamma){ }^{16} \mathrm{O}$ were considered.

VI European Summer School on Experimental Nuclear Astrophysics

Acrireale Italy

September 18-27, 2011

1 Speaker 


\section{Introduction}

Radiative capture reactions involving hydrogen or helium isotopes play an important role in both nucleosynthesis and stellar evolution [1]. Most of the existing data on these reactions have been gained by means of gamma-ray spectroscopy, where the measurements are very difficult in view of the low cross sections and the relatively high background in the detectors. Since some decades a new technique has been exploited, based on the direct detection of the nuclei produced during the reaction using a Recoil Mass Separator (RMS) able to collect the recoil ions keeping the reaction kinematic information, while suppressing the projectile beam emerging from a thin target. A pioneering work was done at Caltech [2], where ${ }^{12} \mathrm{C}(\alpha, \gamma){ }^{16} \mathrm{O}$ was measured using a recoil mass separator and a $\mathrm{NaI}(\mathrm{Tl})$ detection setup. Here the insufficient beam suppression required a coincidence condition between gamma-rays and recoils, thus reducing the advantages of the use of a recoil mass separator. Later started the NABONA (NAples BOchum Nuclear Astrophysics) collaboration between the Institut für Experimentalphysik III of the Ruhr-Universität Bochum, the INFN-Sezione di Napoli and Dipartimento di Scienze Fisiche of the University of Naples Federico II, and for some experiments, the group of the ATOMKI, Debrecen . The aim of this collaboration was to measure the cross section of ${ }^{7} \mathrm{Be}(\mathrm{p}, \mathrm{g})^{8} \mathrm{~B}$. This experiment was performed using a ${ }^{7} \mathrm{Be}$ beam and a windowless hydrogen gas target in combination with a recoil separator [3][4] with sufficient beam suppression and acceptance to detect the recoil without the need of the coincidence condition with gamma-rays. As a follow-up of the NABONA collaboration, a new collaboration between the same groups started, called ERNA, whose aim was to study ${ }^{12} \mathrm{C}(\alpha, \gamma){ }^{16} \mathrm{O}[5][6]$ and ${ }^{3} \mathrm{He}\left({ }^{4} \mathrm{He}, \gamma\right){ }^{7} \mathrm{Be}[7][8]$ using a new recoil separator installed at the 4MV Dynamitron Tandem Laboratorium of the Ruhr-Universität Bochum. At the same time a recoil mass separator named DRAGON [9] was designed and installed at TRIUMF, where several reactions have been studied involving both radioactive [10] and stable beams[11][12][13]. Other similar systems have been developed or are in course of development at different laboratories in Japan and USA [14].

A general review of the field is beyond the scope of the present work. We rather focus on some peculiar aspects of this experimental approach and the description of the ERNA RMS. We consider then the case of ${ }^{12} \mathrm{C}(\alpha, \gamma){ }^{16} \mathrm{O}$ that, besides its enormous interest in nuclear astrophysics, presents the typical difficulties encountered in this kind of experiments.

\subsection{Working principles of RMS's}

In a radiative capture reaction, the fusion of projectile and target nuclei produces a nucleus that recoils in the laboratory system with an average momentum $\mathrm{p}_{\mathrm{r} 0}$ equal to the one of the projectile. In fact, gamma-ray emission determines a change of the momentum of the recoils depending on the gamma ray emission angle. As a result, the trajectories of the recoils lay within a cone centered on the beam axis with an opening angle $\theta=\tan ^{-1}\left(E_{\gamma} /\left(c \cdot p_{r}\right)\right)$, where $E_{\gamma}$ represents the gamma-ray energy and $\mathrm{c}$ the speed of light. Correspondingly, the momentum of the recoils is varied in a momentum range equal to $2 \cdot \mathrm{E}_{\gamma} / \mathrm{c}$ around $\mathrm{p}_{\mathrm{r} 0}$.

The basic principle of a RMS is to determine the reaction yield by means of the direct detection of the recoil ions. In order to make that possible, the reaction is initiated in inverse kinematics, thus maximizing $\mathrm{p}_{\mathrm{r} 0}$. If a thin enough target is used, the kinematically forward focused recoils emerge from the target together with the beam nuclei, whose number exceeds that of recoils by about 10 to 17 orders of magnitude for typical cross sections of astrophysical interest. Therefore an ion optical system consisting of elements such as magnetic dipoles, electrostatic analyzers or 
cross field Wien filters plus some focusing elements is needed to suppress the beam and transport the recoils to an end detector. Since these systems are sensitive to the charge of ions, one of all possible charge states for the recoils must be selected. The finite suppression factor still allows some beam ions to reach the final detector, therefore the possibility of ion identification is desiderable.

The observed yield $Y$ is related to the total reaction cross section $\sigma$ by the following expression:

$$
Y=N_{b} N_{t} \sigma \varepsilon T \Phi\left(q_{r}\right)
$$

where $N_{b}$ is the number of projectiles impinging on the target, whose areal density is $N_{t} . \varepsilon$ is the detection efficiency of the end detector, while $T$ and $\Phi\left(q_{r}\right)$ are, respectively, the transmission and the charge state probability for the selected charge state $q_{r}$.
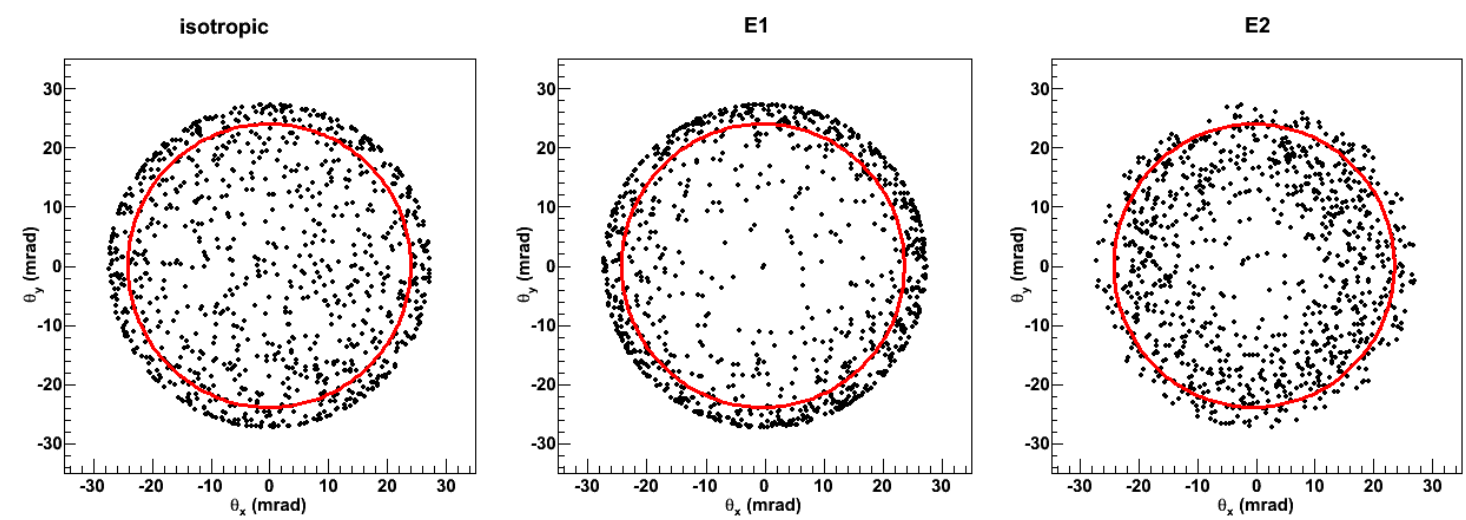

Figure 1 Distribution of recoils in the $\theta_{x}, \theta_{y}$ space for ${ }^{12} C(\alpha, \gamma)^{16} O$ ground state transition at $E=1.0$ MeV. For different $\gamma$-ray angular distributions. Left: isotropic. Center: $\sin ^{2} \theta$. Right: $\sin ^{2} \theta \cos ^{2} \theta$. For reference, a circle shows an angular acceptance of $24 \mathrm{mrad}$. See text for details.

It is obviously crucial to gain a detailed knowledge of all quantities in eq. 1 to extract the total cross section from the observed yield. In most direct experimental approaches to cross section measurements quantities like $N_{p}, N_{t}$, and $\varepsilon$ play a role. Their impact on the cross section measurements is straightforward and generally acknowledged. The case of $\Phi\left(q_{r}\right)$ and $T$ is somewhat different, due to the fact that these parameters are peculiar of RMS, and some aspects of their determination are sometimes not always considered with the due attention.

As regards $\Phi\left(q_{r}\right)$, the target thickness required to operate a RMS is usually not sufficient to reach the charge state equilibrium distribution, and the final recoil charge state distribution is influenced by their charge state distribution at the time of formation. Although some models had some success in specific cases [15][16], there exist no general theory predicting the charge state probability distribution of ions produced in a nuclear reaction. In addition, the different thicknesses traversed by projectile and recoil ions, depending on the reaction coordinate along the targets, result in a different charge exchange before and after the reaction takes place. As a consequence, a post-stripper is often used to reach charge state equilibrium independently of the initial charge state and position of the recoils. Practical designs can be either in form of a windowless gas cell or a foil. Few cases allow alternatively to measure the yield in all possible charge states for the recoils [8]. 
As regards the transmission factor $T$, one has to consider that two contrasting characteristics must be achieved in order to exploit the advantages of this experimental approach. On the one side, the analyzing power must be very high in order to efficiently suppress the projectile beam. On the other side, the acceptance of the system must be large enough to fully collect all recoils, that is $T=1$. Indeed it is worthwhile to note that while the volume in the phase space including all recoils is determined by the gamma-ray energy and the momentum in the laboratory frame, the actual distribution of the recoils within it depends on the angular correlation between the gamma ray emission and the beam direction. An example is given in Figure 1. There the case of ${ }^{12} \mathrm{C}(\alpha, \gamma){ }^{16} \mathrm{O}$ at $\mathrm{E}=1.0 \mathrm{MeV}^{2}$ is considered, where the recoils are emitted in a cone of $27 \mathrm{mrad}$. The plots show the angle formed by the different ion trajectories in the plane perpendicular to the beam axis and an axis symmetric acceptance of $24 \mathrm{mrad}$ is represented by a red circle. Three different angular distributions are considered: isotropic, E1 $\left(\sin ^{2}\right)$ and E2 $\left(\sin ^{2} \cos ^{2}\right)$. Since this acceptance is not sufficient for full transmission of the recoils, the number of detected recoils must be corrected in order to determine the reaction rate and, hence, the cross section. The loss of recoil ions obviously depends on the angular distribution, ranging from $23 \%$ for an E2 transition to $47 \%$ and $66 \%$ for an isotropic and E1 distribution, respectively. The effects of the finite beam size and the interactions of the recoil nuclei with the target material (e.g. straggling) further complicate the calculation of the correction factors and they are not taken into account here. However, it is clear that even a small lack of acceptance may result in a need of a large correction, whose actual value can be calculated only if the angular distribution is known, which is often not the case. As a consequence, measurements in condition of partial acceptance of the recoils may produce inaccurate, and still very precise, results.

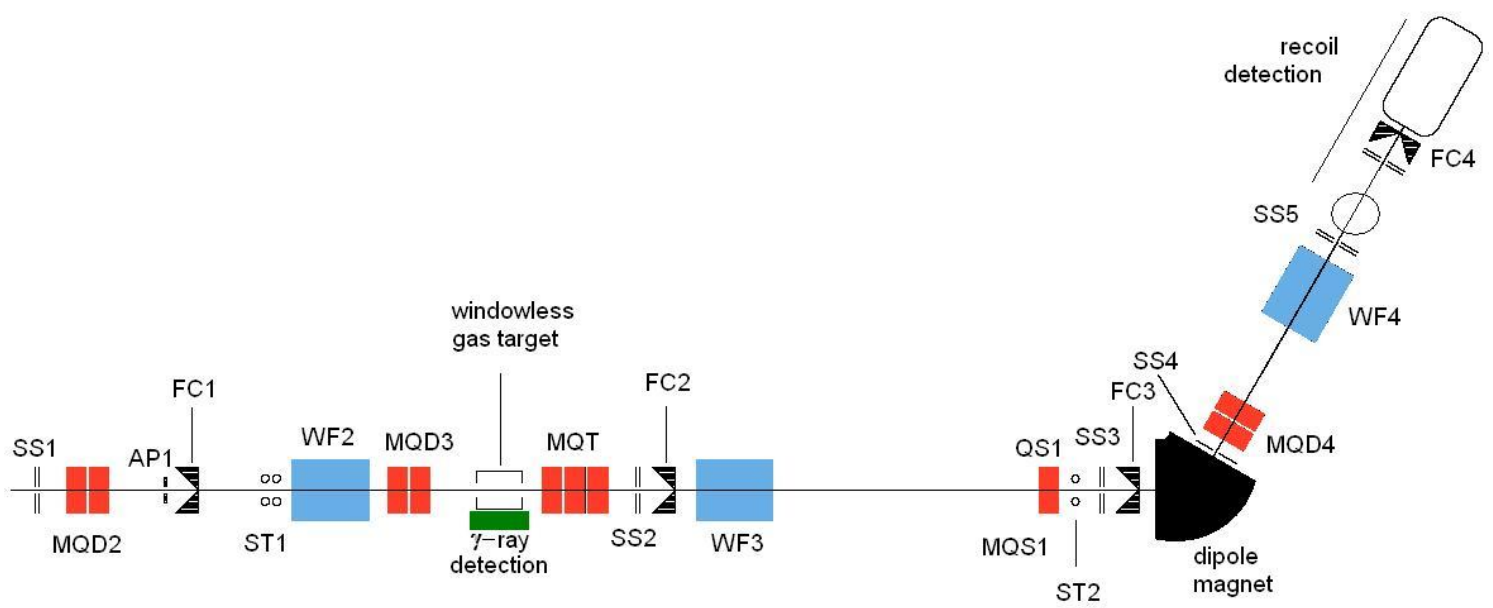

Figure 2 Layout of the RMS ERN. FC=Faraday Cup; ST=Steerer; SS=Slit System; MQT=Magnetic Quadrupole Triplet; MQS=Magnetic Quadrupole Singlet; MQD=Magnetic Quadrupole Dipole; WF $=$ Wien Filter

For that reason, the ERNA collaboration considered mandatory to perform measurements in the condition of full acceptance of the recoils. This in turns requires the acceptance to be known in details. In principle the acceptance can be calculated, if a code describing the mass separator including realistic field maps is available. However, the accuracy of the code needs to be experimentally demonstrated. In the end, it seems more effective to directly measure the acceptance of the separator. This can be done using a deflection unit and varying the beam

\footnotetext{
${ }^{2}$ Energies are in the centre of mass system, if not stated differently.
} 
energy to sample the emittance of the recoils and determine their transmission through the RMS, as reported in [7][17].
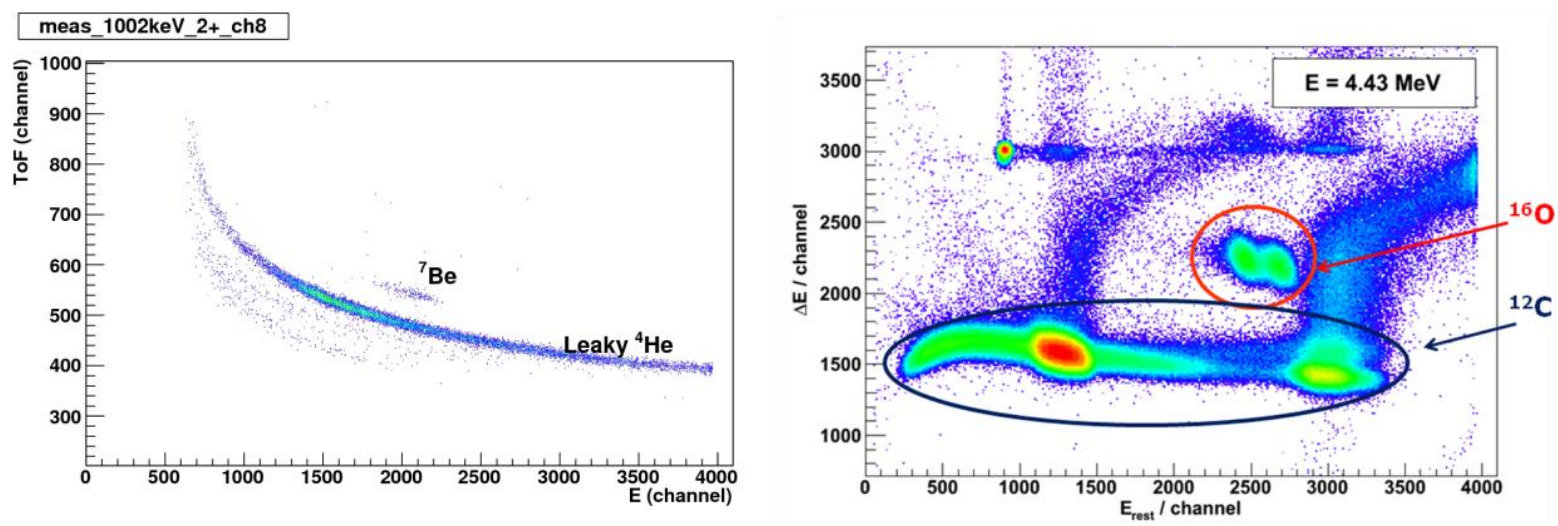

Figure 3 Particle identification with the end detectors of the ERNA recoil mass separator. Left panel shows a Time-Of-Flight vs. E matrix from the measurements of the ${ }^{3} \mathrm{He}\left({ }^{4} \mathrm{He}, \gamma\right)^{7} \mathrm{Be}$ reaction. On the right side a $\Delta E-E$ matrix taken with the ionization chamber telescope from the measurements of ${ }^{12} C(\alpha, \gamma){ }^{16} \mathrm{O}$ is shown.

\subsection{The RMS ERNA}

We will now shortly review how ERNA implements the working principle of a RMS. Figure 2 shows a schematic view of the ERNA setup at the Dynamitron Tandem Laboratory at the Ruhr University Bochum. The experimental setup has been described in details elsewhere [18][19][20]. Briefly, the ion beam emerging from the 4MV Dynamitron tandem accelerator is focused by a quadrupole doublet, filtered by a $52^{\circ}$ analyzing magnet, and guided into the $75^{\circ}$ beam line of ERNA by a switching magnet (these elements are not shown in Figure 2). A quadrupole doublet (MQD2) after the switching magnet is used to focus the beam on the gas target. For the purpose of beam purification, there is one Wien filter (WF1) before the analyzing magnet and one (WF2) between MQD2 and the gas target. The windowless gas target can be operated either in flow-mode or in recirculation. Depending on the pressure in the inner target cell, the target thickness can be as high as about $4 \cdot 10^{17}$ atoms $/ \mathrm{cm}^{2}$. The number of projectiles is determined through the detection of the elastic scattering yield in two collimated silicon detectors located in the target chamber at $75^{\circ}$ from the beam axis. When the gas target is operated in flow mode, an Argon post-target stripping system can be used. After the gas target, the separator consists sequentially of the following elements: a magnetic quadrupole triplet (MQT), a Wien filter (WF3), a magnetic quadrupole singlet (MQS1), a $60^{\circ}$ dipole magnet, a magnetic quadrupole doublet (MQD4), a Wien filter (WF4), and finally a detector for the recoils. Additionally, several steerers (ST), Faraday cups (FC), slit systems (SS), and apertures (AP) are installed along the beam line for setting-up and monitoring purposes. Typical beam suppression factors range from $10^{-10}$ to $10^{-12}$, that is effectively improved by about 3 orders of magnitude using a detector for the recoils able to discriminate them from the leaky projectile beam ions. Different detectors are available for the detection of recoils: a $\Delta \mathrm{E}-\mathrm{E}$ ionization chamber telescope and a TOF-E (Time-Of-Flight vs Energy) setup. The first detector is used at high energies, where the TOF difference between projectile and recoils is small, while the second one is used when the TOF difference between projectiles and recoils is large and a low 
energy threshold is required. Figure 3 shows sample spectra taken with the two detection systems. Furthermore, a NaI $\gamma$-ray detection system [7][5] is installed around the gas target in order to detect gamma-rays in coincidence with the recoils.

ERNA was used to study ${ }^{12} \mathrm{C}(\alpha, \gamma){ }^{16} \mathrm{O}$ and ${ }^{3} \mathrm{He}\left({ }^{4} \mathrm{He}, \gamma\right){ }^{7} \mathrm{Be}$ in Bochum. In section 2 we will discuss the results of the first experiment. Here it is worthwhile to note that in the former case the lowest energy where measurements could be performed was determined by overfocussing of beam ions in the magnetic quadrupole triplet. Thus, a considerable fraction of the beam, being in charge states higher than those selected for the recoils, hit the plates of the first velocity filter, producing an intense leaky beam.

A solution to this problem is the addition of a dipole magnet that selects a single charge state for both recoil and beam ions entering the first lens. The design of this charge state selection magnet is rather complex, since its effective length must be kept as short as necessary in order not to increase the distance of the lens to the target too much. Due to the short length a significant fraction of the magnetic field strength is in the fringe field that needs to be accurately tailored. This solution has been implemented in occasion of the recent movement of ERNA to the Italian laboratory CIRCE (Center for Isotopic research on Cultural and Environmental heritage).

\section{The case of ${ }^{12} \mathrm{C}(\alpha, \gamma)^{16} \mathrm{O}$}

Carbon and oxygen are the main products of helium burning in stars. The reaction ${ }^{12} \mathrm{C}(\alpha, \gamma){ }^{16} \mathrm{O}$, transforming carbon and helium into oxygen, determines the ratio of carbon to oxygen at the end of helium burning. This ratio in turn influences all the further development of a star, such as the production of heavier elements up to iron or the mass of the stellar core prior to supernova explosion. The $\mathrm{C} / \mathrm{O}$ ratio also influences the amount of ${ }^{56} \mathrm{Ni}$, which drives the light curve of supernova I explosions. In $\mathrm{CO}$ white dwarfs constrains can be placed on the internal structure from observations of the stable pulsations during their long cooling phase. Further complication is given by poorly understood mixing scenarios, also influencing the $\mathrm{C} / \mathrm{O}$ ratio at the end of helium burning. Due to this enormous interplay with stellar model ingredients and astrophysical sites, the reaction ${ }^{12} \mathrm{C}(\alpha, \gamma){ }^{16} \mathrm{O}$ is considered a key reaction of nuclear astrophysics, and the reaction rate at astrophysical energies is required to be known within $10 \%$.

At the Gamow peak around $\mathrm{E} \sim 300 \mathrm{keV}$ the cross section is dominated by ground state capture proceeding through two subthreshold resonances with $\mathrm{J}^{\pi}=1^{-}$and $2^{+}$. Those interfere with contributions from higher lying states and the direct capture process. In addition cascade transitions take place. The current estimates of the astrophysical cross section are based on RMatrix analyses, taking into account direct measurements at higher energies $(\mathrm{E}>1 \mathrm{MeV})$, elastic scattering data and the $\beta$-delayed $\alpha$-spectrum of ${ }^{16} \mathrm{~N}$ (providing information on the reduced width of the subthreshold $1^{-}$state).

We will not review the status of our knowledge of this reaction here. We will rather focus on recent measurements that were performed using the RMS technique. The measurements yielded information on the cascade transition through the $6.05 \mathrm{MeV}$ state in ${ }^{16} \mathrm{O}$ [6][13], other cascade transitions [6], and the total capture cross section [5]. 


\subsection{Cascade transition through the 6.05 MeV state}

The capture into the first excited state in ${ }^{16} \mathrm{O}$ at $\mathrm{E}_{\mathrm{x}}=6.05 \mathrm{MeV}$ was not well studied for a long time. The $6.05 \mathrm{MeV}$ state $\left(\mathrm{J}^{\pi}=0^{+}\right)$cannot decay by single photon emission, but will undergo internal pair production in order to decay to the ground state. This makes $\gamma$-spectroscopy of transitions through the $6.05 \mathrm{MeV}$ state complicated, since only primary $\gamma$-rays of relatively low energy can be observed, which are hampered by background in $\gamma$-ray measurements. That problem was overcome by a measurement at the DRAGON facility [13] where $\gamma$-spectra were measured using a BGO detector array in coincidence with ${ }^{16} \mathrm{O}$ recoiling nuclei using a recoil mass separator. Measurements were performed in the energy range $\mathrm{E}_{\mathrm{cm}}=2.2-4.9 \mathrm{MeV}$. The RMatrix analysis of the cross section data assuming a strong E1 contribution yielded $\mathrm{S}_{6.05}(300)=$ $25 \pm 16 \mathrm{keV} \mathrm{b}$ - which would make up about $15 \%$ of the total ${ }^{12} \mathrm{C}(\alpha, \gamma) 16 \mathrm{O}$ cross section at astrophysical energies. One should note, however, that this experiment only reported the angleintegrated strength of the $6.05 \mathrm{MeV}$ transition and no differentiation into E1 and E2 contributions was achieved. As a consequence the assumed E1 contribution had no direct experimental confirmation. In addition, the relatively poor energy resolution did not allow for a clear separation of the energetically close-by $6.13 \mathrm{MeV}$ cascade transition. Transitions into the 6.13 MeV state were excluded in the DRAGON analysis, although its inclusion is reported to occasionally improve the fits to the experimental spectra. Finally, all yields had to be corrected to take into account the insufficient acceptance of the DRAGON RMS. The correction was made based on a GEANT4 simulation of the experimental setup, renormalized to the measured transmission of $\alpha$-particles from a radioactive source. We will come back to this point in section 2.3 .

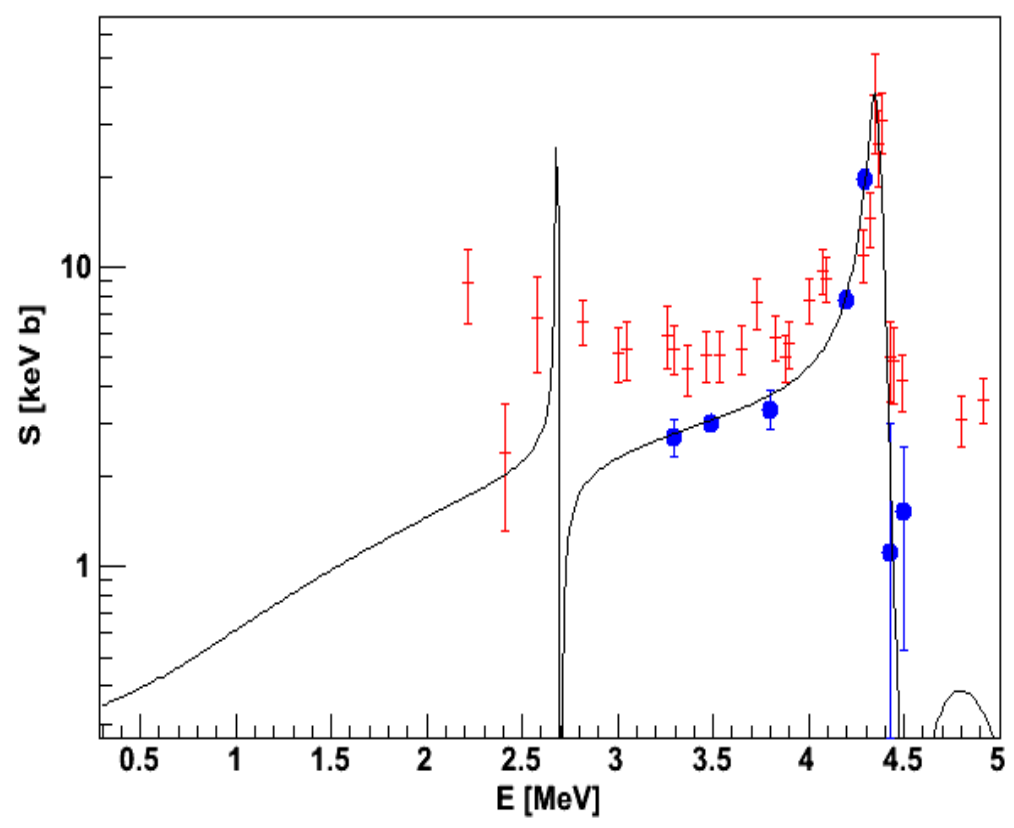

Figure 4: Measurement of the $6.05 \mathrm{MeV}$ transition in ${ }^{12} \mathrm{C}(\alpha, \gamma)^{16} \mathrm{O}$. The experimental points of the DRAGON (red crosses) and ERNA (solid blue circles) experiments vary largely. The R-Matrix fit shown is performed on the ERNA data only and is of pure E2 nature, as determined experimentally.

A new measurement of this transition was performed in the energy range $E=3-5 \mathrm{MeV}$ using the recoil mass separator ERNA and an array of $6 \mathrm{NaI} \gamma$-ray detectors [6]. In that experiment, the E1 and E2 contributions to the cross section could be determined. The E1 contribution was 
found to be negligible. All cascade and ground state transitions were considered in the analysis of the $\gamma$-ray spectra, based on fits of GEANT4 spectra. In particular the transition into the 6.13 $\mathrm{MeV}$ state was clearly identified. As a result of its inclusion, the cross section for this transition turns out to be generally lower than the previous estimate. The results of the two measurements and the new R-Matrix calculation are shown in Figure 4. The R-Matrix analysis from [6] yields values of $S_{6.05}(300)<1 \mathrm{keV} b$, thus being of no relevance on the astrophysical scale.

\subsection{Other cascade transitions}

The ERNA $\gamma$-ray measurement also provides data on the cascade transitions into the 6.92 and 7.12 MeV states and supplements existing measurements of these cascade transitions. Previous measurements are present in an energy range of $1.5 \mathrm{MeV}$ to $3 \mathrm{MeV}$ [22][23][24]. Among those measurements, the work of Kettner et al. is often cited as a measurement of the $6.92 \mathrm{MeV}$ transition - as considered in the corresponding publication. Data in that work have been obtained in coincidence of two 4"x4" NaI detectors in close geometry and therefore the cross sections given in Kettner et al. must include both the $6.92 \mathrm{MeV}$ and the $7.12 \mathrm{MeV}$ transitions. Redder et al. and Kunz et al. could separate the two transitions and reported the strength of both. In case of the work [24] the cascade data are accessible from the Ph.D. thesis [25]. In general a poor agreement among the different data sets is found. A possible explanation is given in [25] with the presence of background lines in the $\gamma$-ray spectra.
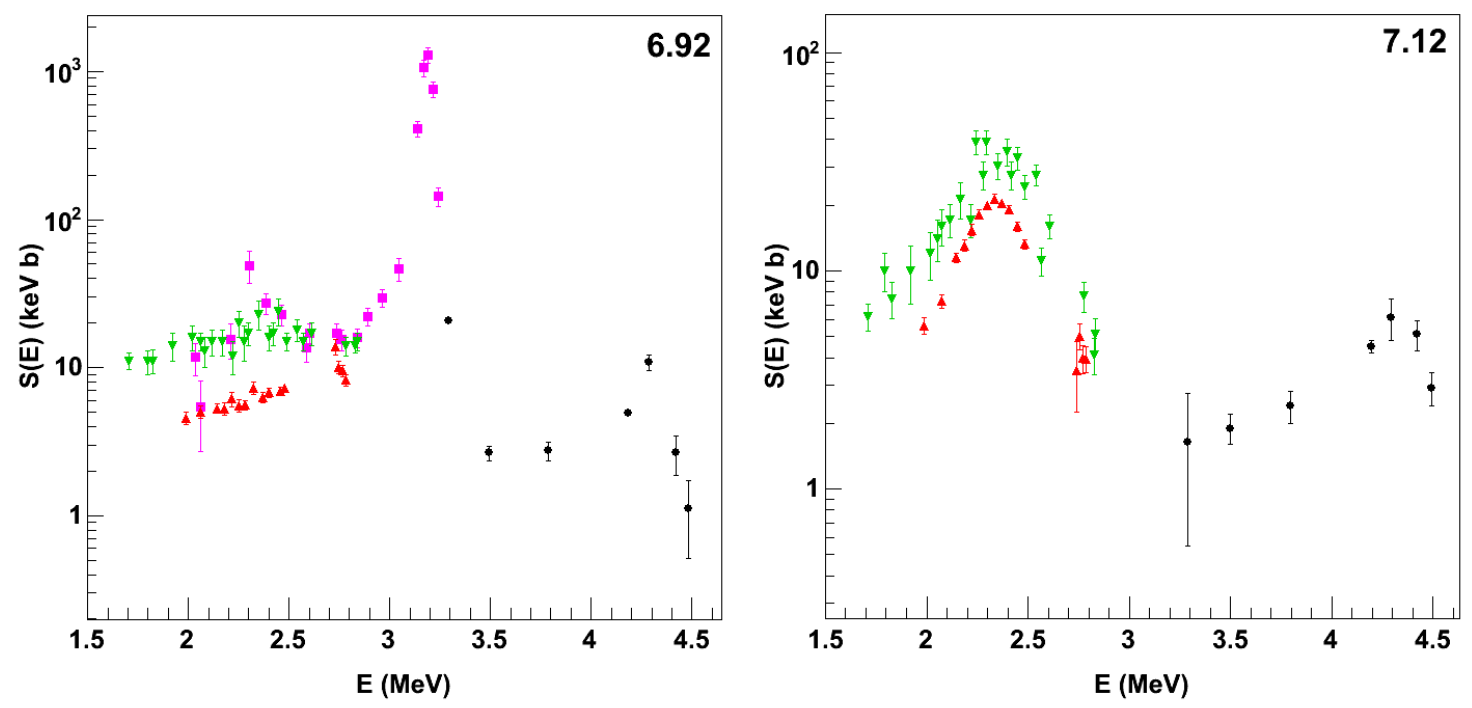

Figure 5: Cascades transitions into the 6.92 and $7.12 \mathrm{MeV}$ states of ${ }^{16} \mathrm{O}$ from ${ }^{12} \mathrm{C}(\alpha, \gamma){ }^{16} \mathrm{O}$. Data are taken from Kunz et al. (solid red triangles-up, [24][25]), Redder et al. (solid green triangles-down, [22]), Kettner et al. (full magenta squares, [23]) and Schürmann et al. (black dots, [6]). The seemingly good agreement between [22] and [23] is invalidated by the fact that the latter measurement is the sum of the 6.92 and $7.12 \mathrm{MeV}$ transitions, rather than the $6.92 \mathrm{MeV}$ transition alone.

With the ERNA data we now do have a new source of information on these transitions at higher energies. Included in R-Matrix fits the new data will allow for a better understanding of the cascade transitions not only in the range of earlier $\gamma$-ray experiments, but also in the extrapolation of these transitions to astrophysical energies. Figure 5 summarizes the status of the 6.92 and $7.12 \mathrm{MeV}$ cascade transition measurements. 


\subsection{Total cross section}

Recoil mass separator measurements also yield a different source of information by counting recoils without $\gamma$-ray coincidences. These measurements of the total cross sections benefit from the huge efficiency of the separator, while removing uncertainties from the absolute efficiency calibration and background contaminations found in $\gamma$-ray measurements. In the ERNA experiment the total cross section of ${ }^{12} \mathrm{C}(\alpha, \gamma){ }^{16} \mathrm{O}$ was measured in the energy range of $2-5$ $\mathrm{MeV}$ [5] with typical statistical uncertainties of 3\%. In a simultaneous fit of all $\gamma$-ray transitions the total cross section data are used to determine the sum of the single transitions reported in many different $\gamma$-ray experiments. Figure 6 compares the ERNA total cross section data to the sum of $\gamma$-ray data from [22][23][24], the only $3 \gamma$-ray experiments where all relevant transitions were concurrently measured. In the case of [23] it was assumed that the $6.92 \mathrm{MeV}$ transition reflects the sum of 6.92 and $7.12 \mathrm{MeV}$ transitions, as discussed above. We also compare with the total cross section as measured in the DRAGON experiment [13], which is reported in the corresponding Ph.D. thesis [21]. The total cross section seems to agree well with the ERNA data on a wide energy range, however with some remarkable deviations around the $2.4 \mathrm{MeV} 1^{-}$ and $4.4 \mathrm{MeV} 2^{+}$resonances. The $1^{-}$resonance is essentially absent in the DRAGON measurement. Since in this region much larger acceptances are required than DRAGON actually has, the observed discrepancy is an indication of an inaccurate correction for the missing acceptance, which might possibly also be the case for the $\gamma$-ray measurements of [13].

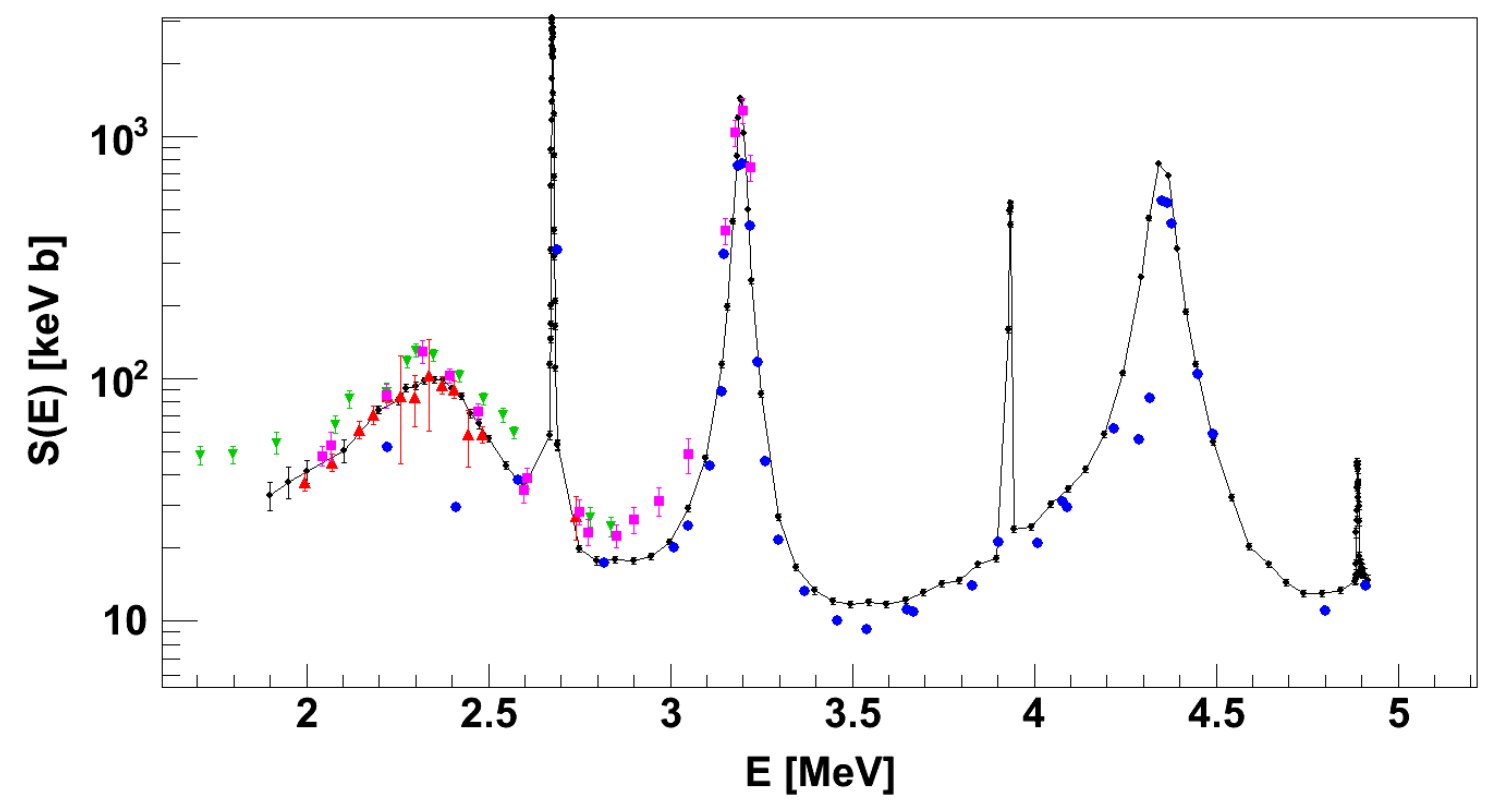

Figure $6{ }^{12} \mathrm{C}(\alpha, \gamma){ }^{16} \mathrm{O}$ total cross section as measured by the RMS experiments ERNA (black dots with line to guide the eye, [5]) and DRAGON (solid blue circles, [21]) compared to the sum of all $\gamma$-ray transitions from the measurements of Kunz et al. (solid red triangles-up, [24][25]), Kettner et al. (solid magenta squares, [23]) and Redder et al. (solid green triangles-down, [22]). Good agreement is found between between ERNA and Kunz et al., while the DRAGON data points show larger deviations around the resonances at 2.4 and $4.4 \mathrm{MeV}$. 


\section{Summary and Outlook}

The results obtained so far show that RMS are powerful tools for studying radiative capture reactions of interest in nuclear astrophysics. This approach offers the advantage of a nearly background free, high precision measurement and a strong control of systematic effects, if the relatively complex experimental setup and procedures are accurately characterized.

In particular, we presented the case of ERNA and the study of ${ }^{12} \mathrm{C}(\alpha, \gamma){ }^{16} \mathrm{O}$. New information could be obtained on cascade and ground state transitions as well as on the total cross section. We compared the ERNA data with previous measurements. Particularly interesting is the comparison with the results of another experiment performed using the RMS Dragon, where we found significant discrepancies compared to the ERNA data. We showed that the discrepancies are probably due to systematic uncertainties affecting the measurement at DRAGON arising from the poor angular and energy resolution of the $\gamma$-ray detector array and the insufficient acceptance of the separator. These kind of uncontrolled systematic effects obstruct the way toward a precise and accurate determination of the stellar rate of ${ }^{12} \mathrm{C}(\alpha, \gamma){ }^{16} \mathrm{O}$. In order to improve this determination, data must be selected according to rigorous criteria and documented systematic uncertainties of the included data sets, in particular the absolute normalization, must be treated in the fitting procedure. Combining such improvements in the data handling with the new recoil separator data leads to R-Matrix descriptions of the ${ }^{12} \mathrm{C}(\alpha, \gamma){ }^{16} \mathrm{O}$ reaction approaching the precision required by stellar models [26].

\section{References}

[1] C.E. Rolfs and W.S. Rodney, Cauldrons in the Cosmos, University of Chicago Press (1988)

[2] R. M. Kremer et al., Phys. Rev. Lett. 60, 1475 (1988)

[3] L. Gialanella et al., Eur. Phys. J. A 7, 303-305 (2000)

[4] L. Gialanella et al., Nucl. Instr. Meth. A 376 174-814 (1996)

[5] D. Schürmann et al., Eur. Phys. J. A 26 (2005) 301

[6] D. Schürmann et al., Phys. Lett. B 703 (2011) 557

[7] A. Di Leva et al., Nucl. Instr. Meth. A 595 (2008) 381-390

[8] A. Di Leva et al., Phys. Rev. Lett. 102, 232502 (2009)

[9] D. A. Hutcheon et al., Nucl. Instr. Meth. A 498 (2003) 190-210

[10] S. Bishop et al., Phys. Rev. Lett. 90, 162501 (2003)

[11] C. Vockenhuber et al., Phys. Rev. C 76035801 (2007)

[12] U. Hager et al., Phys. Rev. C 84, 022801(R) (2011)

[13] C. Matei et al., Phys. Rev. Lett. 97 (2006) 242503

[14] M. Couder et al., Nucl. Instr. Meth. A 587 (2008) 35-45

[15] J. Zylberberg et al., Nucl. Instr. Meth. B, 254 (2007)

[16] D. Schürmann, Ph.D. Thesis, Bochum 2007, http://www-brs.ub.ruhr-uni-bochum.de/netahtml/HSS/Diss/SchuermannDaniel/diss.pdf 
[17] D. Schürmann et al., Nucl. Instr. Meth. A 531 (2004) 428

[18] D. Rogalla et al., Eur. Phys. J. A 6 (1999) 471-477

[19] D. Rogalla et al., Nucl. Instr. Meth. A 513(2003) 573-578

[20] L. Gialanella et al., Nucl. Instr. Meth. A 522(2004) 432-438

[21] C. Matei, Ph.D. Thesis Ohio University 2006, http://rave.ohiolink.edu/etdc/view?acc_num=ohiou1159463892

[22] A. Redder et al., Nucl. Phys. A 462 (1987) 385

[23] K.U. Kettner et al., Z. Phys. A 308 (1982) 73-94

[24] R. Kunz et al., Phys. Rev. Lett. 86 (2001) 3244

[25] R. Kunz, ${ }^{12} \mathrm{C}(\alpha, \gamma){ }^{16} \mathrm{O}$ - Die Schlüsselreaktion im Heliumbrennen der Sterne, Ph.D. thesis, Universität Stuttgart, Germany, 2002

[26] D.Schürmann, L.Gialanella, R. Kunz and F. Strieder, submitted to Phys. Lett. B 